University of Nebraska - Lincoln

DigitalCommons@University of Nebraska - Lincoln

Faculty Publications from the Department of Engineering Mechanics

Mechanical \& Materials Engineering,

Department of

2-1994

\title{
Inhomogeneous anisotropic percolation: Two-dimensional numerical threshold analysis
}

\author{
Yuris A. Dzenis \\ University of Nebraska-Lincoln, ydzenis@unl.edu \\ S. P. Joshi \\ University of Texas at Arlington, Arlington, Texas
}

Follow this and additional works at: https://digitalcommons.unl.edu/engineeringmechanicsfacpub

Part of the Mechanical Engineering Commons

\footnotetext{
Dzenis, Yuris A. and Joshi, S. P., "Inhomogeneous anisotropic percolation: Two-dimensional numerical threshold analysis" (1994). Faculty Publications from the Department of Engineering Mechanics. 38. https://digitalcommons.unl.edu/engineeringmechanicsfacpub/38
}

This Article is brought to you for free and open access by the Mechanical \& Materials Engineering, Department of at DigitalCommons@University of Nebraska - Lincoln. It has been accepted for inclusion in Faculty Publications from the Department of Engineering Mechanics by an authorized administrator of DigitalCommons@University of Nebraska - Lincoln. 


\title{
Brief Reports
}

Brief Reports are accounts of completed research which, while meeting the usual Physical Review standards of scientific quality, do not warrant regular articles. A Brief Report may be no longer than four printed pages and must be accompanied by an abstract. The same publication schedule as for regular articles is followed, and page proofs are sent to authors.

\section{Inhomogeneous anisotropic percolation: Two-dimensional numerical threshold analysis}

\author{
Yu. A. Dzenis and S. P. Joshi \\ Center for Composite Materials, Department of Mechanical and Aerospace Engineering, \\ University of Texas at Arlington, Arlington, Texas 76019 \\ (Received 9 August 1993; revised manuscript received 12 October 1993)
}

\begin{abstract}
Monte Carlo simulations of percolation on a square lattice with anisotropic inhomogeneous probability distribution are reported. Finite-size scaling is used for data analysis. As inhomogeneity increases, the critical probability decreases; whereas the correlation-length exponent remains, within computation errors, the same as in classical two-dimensional percolation.
\end{abstract}

The problem of anisotropic percolation has been a subject of many investigations. Anisotropy can be induced by various factors. The percolation of systems with anisotropy due to the anisotropy of properties ${ }^{1-3}$ or shapes $^{3-6}$ of single-component regions as well as due to the orientational dependence of connectivity rules $^{7,8}$ has been studied theoretically and experimentally.

In our recent research on stochastic damage evolution in laminates, ${ }^{9-12}$ another type of anisotropy in random systems was perceived; the one due to a spatial inhomogeneity of the probability of crack formation in plies. In general, there are several types of cracks in each ply interacting with each other. The probability of crack formation depends on the orientation of the ply in a laminate and the applied load. For layups with alternate ply orientation, simple periodic probability distribution is observed. As a first approximation, assume that microcracks of different types are similar for percolation analysis. The problem of crack formation, accumulation and propagation through the thickness of a laminate can be considered as an anisotropic site percolation in two dimensions with the probability of crack formation in plies equal to the sum of probabilities of damages of different types. In developing statistical models for damage in solids, ${ }^{13}$ cluster statistics are of primary importance, especially size, shape, and orientation statistics. However, it is necessary to study the critical behavior of the system $^{14,15}$ before investigating the cluster properties. This paper presents an analysis of the effects of inhomogeneity on the percolation threshold and correlation-length exponent in two dimensions using Monte Carlo simulations and the finite-size scaling technique.

A model consisting of a simple square lattice of side $b$ containing rows with alternating probabilities of occupancy of sites $p_{1}$ and $p_{2}$ is studied. The average probability of occupancy of a site on the lattice is $p=\left(p_{1}+p_{2}\right) / 2$. The inhomogeneity parameter is $c=p_{1} / p_{2}$. Due to the symmetry of the problem we can arbitrarily choose $p_{1} \leq p_{2}$, then $c \in[0,1]$. Let us define $p_{H}$ as a probability $p$ at percolation in the horizontal direction (along the rows), and $p_{V}$ as a probability at percolation in the vertical direction. It is clear that for $c \neq 1$, the system exhibits anisotropic percolation on finite lattices, but percolation thresholds $p_{H}, p_{V}$ approach an isotropic limit $\left(p_{c}(c)\right.$ when the lattice size increases.

For any finite lattice, percolation thresholds $p_{V}, p_{H}$ are random and their distributions $L\left(c, b: p_{V}\right)$ and $L\left(c, b: p_{H}\right)$ are narrower for larger lattices. These distributions are expected to be roughly Gaussian. According to finite-size scaling considerations, ${ }^{16,17}$ both mean $\langle p\rangle$ and deviation $\sigma=\left\langle(p-\langle p\rangle)^{2}\right\rangle^{1 / 2}$ of distributions for all $c$ 's scale for sufficiently large $b$ as

$$
\left|p_{c}-\langle p\rangle\right| \sim b^{-1 / v}
$$

TABLE I. Means and deviations of thresholds distributions in two directions for inhomogeneous percolation models $c=0.4$ and $c=0.2$.

\begin{tabular}{|c|c|c|c|c|c|}
\hline \multicolumn{6}{|c|}{$c=0.4$} \\
\hline$b$ & $N$ & $\left\langle p_{V}\right\rangle$ & $\sigma_{V}$ & $\left\langle p_{H}\right\rangle$ & $\sigma_{H}$ \\
\hline 10 & 8000 & 0.5758 & 0.0695 & 0.5158 & 0.0651 \\
\hline 20 & 4000 & 0.5684 & 0.0435 & 0.5266 & 0.0423 \\
\hline 40 & 2000 & 0.5632 & 0.0262 & 0.5370 & 0.0255 \\
\hline 80 & 1000 & 0.5581 & 0.0154 & 0.5426 & 0.0149 \\
\hline 160 & 759 & 0.5551 & 0.0089 & 0.5457 & 0.0089 \\
\hline 320 & 199 & 0.5532 & 0.0053 & 0.5472 & 0.00581 \\
\hline 640 & 51 & 0.5526 & 0.0035 & 0.5490 & 0.0029 \\
\hline 1280 & 12 & 0.5512 & 0.0014 & 0.5501 & 0.0015 \\
\hline \multicolumn{6}{|c|}{$c=0.2$} \\
\hline 40 & 2000 & 0.5447 & 0.0198 & 0.4964 & 0.0209 \\
\hline 80 & 1000 & 0.5348 & 0.0124 & 0.5059 & 0.0128 \\
\hline 160 & 891 & 0.5294 & 0.0078 & 0.5115 & 0.0074 \\
\hline 320 & 241 & 0.5255 & 0.0044 & 0.5150 & 0.0044 \\
\hline 640 & 69 & 0.5229 & 0.0027 & 0.5170 & 0.0028 \\
\hline 1280 & 17 & 0.5225 & 0.0009 & 0.5191 & 0.0014 \\
\hline
\end{tabular}




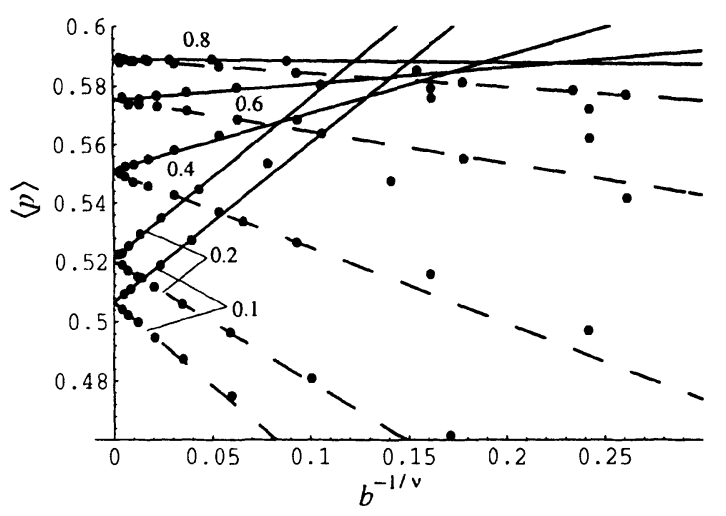

FIG. 1. Linear least-square fits for deviations $\sigma(b)$ as a function of the lattice size; $\sigma_{H}$ (dash), $\sigma_{v}$; (solid) $c=0.1,0.2,0.4,0.6$, and 0.8 .

$$
\sigma \sim b^{-1 / v}
$$

where $v$ is a correlation-length exponent. Calculating $L\left(c, b: p_{V}\right)$, and plotting $\sigma(b)$ in double logarithmic coordinates for a number of lattice sizes $b,(v)$ can be obtained from (2) as a slope of the curve. Once $v$ is known, extrapolating $\langle p\rangle\left(b^{-1 / v}\right)$ to $b^{-1 / v}=0$ gives an estimate of $p_{c}$.

Unlike homogeneous percolation where the maximum probability $p$ on a lattice is equal to 1 and the percolation threshold can always be achieved by increasing $p$, in the case of inhomogeneous percolation, maximum $p$ is $(1+c) / 2$. At this probability $p_{2}=1$ and $p_{1}=c \leq 1$. Hence, for a finite lattice there exists a nonzero probability of the absence of the percolation path in the vertical direction (across the rows). This probability is higher for smaller lattice sizes and lower $c$ 's (higher inhomogeneity levels). The probability of the existence of a percolation path in the $V$ direction at maximum $p=(1+c) / 2$ is given by

$$
p_{V_{e}}=\left[1-(1-c)^{b}\right]^{b / 2} .
$$

Numerical analysis of distributions $L\left(c, b: p_{V}\right)$ shows that when $p_{V_{e}}$ is sufficiently lower than 1 , they become

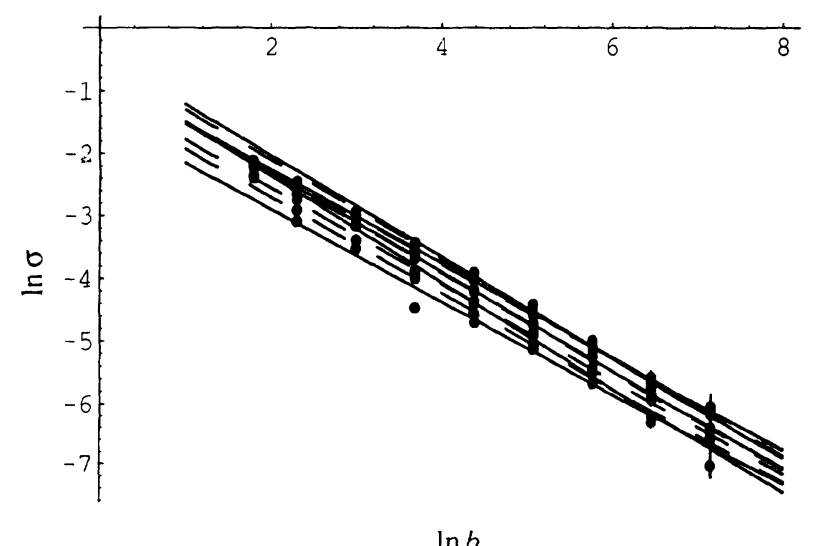

FIG. 2. Linear least-square fits for $\langle p\rangle$ as a function of $\left(b^{-1 / v}\right)$ for several inhomogeneity parameters $c ; p_{H}$ (dash) $p_{V}$ (solid).
TABLE II. Summary for correlation-length exponents and percolation thresholds at various inhomogeneity levels.

\begin{tabular}{lccc}
\hline$c$ & $v_{V}$ & $v_{H}$ & $p_{c}$ \\
\hline 1 & 1.448 & 1.359 & 0.5927 \\
0.8 & 1.235 & 1.264 & 0.5888 \\
0.6 & 1.334 & 1.336 & 0.5750 \\
0.4 & 1.265 & 1.262 & 0.5506 \\
0.2 & 1.379 & 1.362 & 0.5206 \\
0.1 & 1.357 & 1.310 & 0.5064 \\
\hline
\end{tabular}

considerably non-Gaussian (one sided). It is clear that larger lattice sizes are required for smaller inhomogeneity parameters. The smallest inhomogeneity parameter used for numerical simulation is 0.1 . A minimum lattice size to be employed in calculations is determined from (3) as one for which $p_{V_{e}} \geq 0.95$. Calculations are performed by a method similar to Ref. 18 on the Cray Y-MP computer. The computer code is written in $C$ language and utilizes a dynamic memory allocation.

The calculations are checked for a homogeneous case $c=1$. The following range of $b$ is investigated: $10,20,40$, $80,160,320$, and 640 . Obtained threshold distributions are checked for normality by calculating the third and fourth moments. Comparisons with Ref. 19 show that the calculated values fall within the $95 \%$ confidence interval for normal distributions. Comparisons with numerical data ${ }^{20}$ show good agreement.

Distributions $L\left(c, b: p_{V}\right)$ and $L\left(c, p: p_{H}\right)$ are calculated for $c=0.8,0.6,0.4,0.2$, and 0.1. An additional lattice size of 1280 is employed in the analysis. Calculated means and deviations of threshold distributions for $c=0.4$ and 0.2 are listed in Table $I$ for future reference. Figure 1 shows dependences of $\langle p\rangle$ on $b^{-1 / v}$. Error bars for threshold means estimated by $\Delta\langle p\rangle=\sigma / \sqrt{N}$ do not exceed data point size in Fig. 1. Lines represent linear least-square fits for larger lattices. It is seen that linear approximations for percolation threshold in two directions tend toward one limit for each value of $c$ examined. Their intersection with the ordinate axis gives a "true" value of $p_{c}$ for given $c$.

The correlation-length exponent $v$ for plotting Fig. 1 is

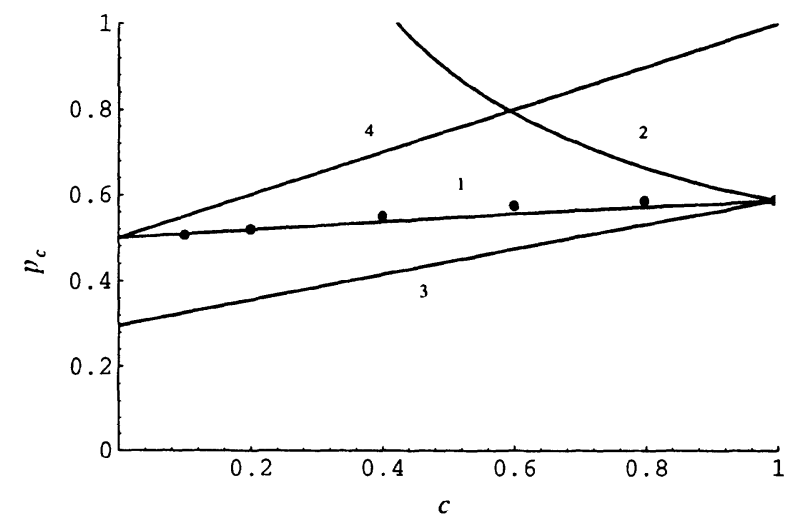

FIG. 3. Variation of $p_{c}$ with $c$ : (points) numerical experiments; (1) linear variation between $p_{c}(0)$ and $p_{c}(1) ;(2,3) p_{V}, p_{H}$ for joined infinite semiplanes, respectively; (4) maximum $p=(1+c) / 2$. 
obtained from Fig. 2. Figure 2 shows the percolation threshold deviations plotted as functions of $b$. The error bars for $\sigma$ are calculated by

$\Delta \sigma=\frac{1}{2 \sigma \sqrt{N}} \sqrt{\left\langle\left(p_{i}-\langle p\rangle\right)^{4}\right\rangle-\left\langle\left(p_{i}-\langle p\rangle\right)^{2}\right\rangle^{2}}$.

Error bars are too small to be visible in Fig. 2 except for large lattice sizes. Correlation-length exponents obtained by linear least-square fits at higher $b$ and slope estimations given in Table II. Within experimental error they coincide with the classical value $v=4 / 3$ for two dimensions, which is consistent with the concept of universality confirmed for other anisotropic systems. $1,5,8$

A summary of estimates of $p_{c}(c)$ is shown in Table II.
It is seen that as inhomogeneity increases, percolation threshold decreases. The limit for $p_{c}$ when $c \rightarrow 0$ is 0.5 . Experimentally obtained dependence $p_{c}(c)$ is not a linear variation between the limits $p_{c}(0)=0.5$ and $p_{c}(1)$ $=0.5927$, as shown in Fig. 3. Figure 3 also shows bounds for $p_{V}, p_{H}$ calculated as an average probability at percolation in two directions for a model consisting of two half planes with occupancy probabilities $p_{1}, p_{2}$, such that $p_{1} / p_{2}=c$.

This work is partially funded by the Army Research Office, Grant No. DAAL03-89-G-0090. Computer time is provided by the University of Texas System Center for High Performance Computing.
${ }^{1}$ C. J. Lobb, D. J. Frank, and M. Tinkham, Phys. Rev. B 23, 2262 (1981).

${ }^{2}$ A. K. Sarychev and A. P. Vinogradoff, J. Phys. C 12, L681 (1979).

${ }^{3}$ B. I. Shklovskii, Phys. Status Solidi B 85, K111 (1978).

${ }^{4}$ F. Carmona, F. Barreau, P. Delhaes, and R. Canet, J. Phys. (Paris) Lett. 41, L531 (1980).

${ }^{5}$ J. Boissonade, F. Barreau, and F. Carmona, J. Phys. A 16, 2777 (1983).

${ }^{6}$ L. N. Smith and C. J. Lobb, Phys. Rev. B 20, 3653 (1979).

${ }^{7}$ R. Blanc, C. D. Mitescu, and G. Threvenot, J. Phys. (Paris) Colloq. 41, C3-387 (1980).

${ }^{8}$ S. Redner and H. E. Stanley, J. Phys. A 12, 1267 (1979).

${ }^{9}$ Yu. A. Dzenis, S. P. Joshi, and A. E. Bogdanovich, in $33 r d$ AIAA/ASME/ASCE/AHS/ASC Structures, Structural Dynamics and Material Conference, 1992 (AIAA, Washington, D.C., 1992), Part 5, pp. 2887-2897.

${ }^{10}$ Yu. A. Dzenis, S. P. Joshi, and A. E. Bogdanovich, in 7th ASC Technical Conference on Composite Materials, 1992 (Technomic, Lancaster, 1992), pp. 1084-1093.

${ }^{11}$ Yu. A. Dzenis, S. P. Joshi, and A. E. Bogdanovich, AIAA J. (to be published).
${ }^{12} \mathrm{Yu}$. A. Dzenis and S. P. Joshi, in 34th $A I A A / A S M E /$ ASCE/AHS/ASC Structures, Structural Dynamics and Material Conference, 1993 (AIAA, Washington, D.C., 1993).

${ }^{13}$ Statistical Models for the Fracture of Disordered Media, edited by H. J. Herrmann and S. Roux (North-Holland, Amsterdam, 1990).

${ }^{14}$ D. Stauffer, Phys. Rep. 54, 1 (1979).

${ }^{15}$ J. Kertesz, D. Stauffer, and A. Coniglio, in Percolation Structures and Processes (Israel Physical Society, Jerusalem, 1983), pp. $121-147$.

${ }^{16}$ M. E. Levinshtein, B. I Shklovskii, M. S. Shur, and A. L. Efros, Zh. Eksp. Teor. Fiz. 69, 386 (1975) [Sov. Phys. 42, 197 (1975)].

${ }^{17}$ J. Roussenq, J. Clerc, G. Giraud, E. Guyon, and H. Ottavi, J. Phys. (Paris) Lett. 37, L99 (1976).

${ }^{18}$ A. S. Skal and B. I. Shklovskii, Fiz. Tekh. Poluprovodn. 8, 1586 (1974) [Sov. Phys. Semicond. 8, 1029 (1975)].

${ }^{19}$ Biometrika Tables for Statisticians, edited by E. S. Pearson and H. O. Hartley (Cambridge University Press, Cambridge, 1966). Vol. 1.

${ }^{20}$ P. J. Reynolds, H. E. Stanley, and W. Klein, Phys. Rev. B 21, 1223 (1980). 